\title{
Nivel de infección por estróngilos digestivos de padrotes ovinos y de sus hijas como criterio de selección de ovinos helmintorresistentes
}

\author{
Levels of infection by gastrointestinal parasites of \\ sire and theirs daughters as criteria for selection of \\ helminth resistant sheep
}

Fecha recepción: 28 de julio de 2013

Fecha Aprobación: 29 de agosto de 2013
Gustavo Adolfo Morales-Contreras ${ }^{1}$, Espartaco Sandoval' ${ }^{2}$, Luz Arelis Pino de Morales ${ }^{3}$, Jairo Morales ${ }^{4}$, Delia Jiménez ${ }^{5}$

\section{RESUMEN}

Mediante el análisis multivariado de correspondencia lineal simple, se evaluó la asociación entre los niveles de infección por nemátodos gastrointestinales, expresados en recuentos de huevos por gramo de heces (h.p.g.) y categorizados como Negativo, Leve, Moderado y Alto, tanto de las hijas agrupadas por padrote, como de los padrotes. El padrote de raza Bergamasca se asoció con hijas con niveles de infección leves o moderados, mientras que uno de los padrotes de la raza Barbados Barriga Negra, el BBN 1, se asoció con hijas negativas a la infección por estróngilos digestivos, y el otro (BBN 2), con niveles altos de infección de sus hijas. Al analizar cada padrote con sus respectivos niveles de infección se ve que el padrote Bergamasca se asocia con niveles

\begin{abstract}
The relationship between levels of infection with gastrointestinal nematodes expressed in eggs per gram counts (e.p.g) and categorized as negative, light, moderate and heavy, were analyzed by multivariate analysis of simple linear correspondence and the following results were obtained: The Bergamasca's daughters were associated with light to moderate level of infection, while in the case of the Barbados Black Belly 1 (BBN 1) daughters, it was found an association with a negative level of infection. The daughters of the Barbados Black Belly 2 (BBN 2) were associated with heavy level of infection. When analyzing each sire breed with their infection levels, it was found that: the Bergamasca sire was associated with a moderate levels of
\end{abstract}

Ph.D. Instituto Nacional de Investigaciones Agrícolas, Centro Nacional Investigaciones Agropecuarias (MaracayVenezuela). Correo electrónico: gustavomoralesc@gmail.com

Ph.D. Instituto Nacional de Investigaciones Agrícolas (Yaracuy-Venezuela). Correo electrónico: esandoval@inia.gob.ve Ph.D. Universidad de Los Andes (Trujillo-Venezuela).

Ejercicio libre de la profesión

Ejercicio libre de la profesión 
moderados de infección, mientras que el Barbados Barriga Negra 1 (BBN 1) lo hizo con niveles de infección que oscilan entre leves y moderados. El padrote Barbados Barriga Negra 2 (BBN 2) mostró buena correspondencia con niveles de infección altos. Los resultados obtenidos evidencian una buena asociación entre los niveles de infección observados en los padrotes y el de cada lote de hijas separadas por cada padre. Conociendo que el recuento de h.p.g es una expresión fenotípica de resistencia frente a la infección por estróngilos digestivos, característica de índole genética y, por consiguiente heredable, concluimos que el padrote que expresó mejor capacidad helmintorresistente fue el BBN 1.

Palabras clave: H.P.G., Análisis de correspondencia, Ovinos, Helmintorresistente. infection, while the Barbados Black Belly 1 (BBN 1) showed infections levels oscillating from light to moderate. The sire Barbados Black Belly 2 (BBN 2) showed a good correspondence with heavy levels of infection. In conclusion, knowing the e.p.g count is a phenotypic expression of resistance to parasitic gastrointestinal infection, that is genetic in nature and therefore heritable. the selection of sire Barbados Black Belly 1(BBN 1) is discussed and proposed. The sire with the highest capacity Helminth Resistant was the BBN1

Key Words: E.p.g., Correspondence Analysis, Helminth Resistant, Sheep, Sire. 


\section{INTRODUCCIÓN}

La helmintorresistencia ha sido definida como la capacidad de resistir al establecimiento y posterior desarrollo de las larvas infestantes (L3) ingeridas por el hospedador, así como de disminuir la velocidad de desarrollo de los diferentes estados larvarios y de su evolución hacia adultos y de afectar negativamente la tasa de sobrevivencia de los parásitos adultos, lo cual se traduce en que los animales resistentes o no albergan parásitos o, si están parasitados, las cargas por ellos soportadas son bajas y las hembras parásitas presentes tienen un nivel de oviposturas bajo $(1,2)$.

El aumento de la resistencia de los animales reduce la contaminación de las pasturas y, por consiguiente, disminuye la disponibilidad de larvas infestantes para los animales susceptibles del rebaño (1).

En los ovinos existe amplia evidencia sobre la posibilidad de explotar la variación genética en la resistencia a la infección por nemátodos gastroentéricos como criterio de selección de los reproductores (3). Según Baker (4), la variación genética relacionada con la resistencia a las enfermedades es de naturaleza poligénica, y en el caso específico de las helmintosis gastroentéricas en ovinos se dispone de interesantes resultados; por ejemplo, en la zona subhúmeda de Kenya, dicho autor (4) señala que los corderos de la raza Red Massai son más resistentes a los endoparásitos que los de la raza Dorper, y en infecciones naturales multiespecíficas se demostró que los ovinos de raza Merino D'Arles son más resistentes a la infección por estróngilos digestivos que los Romanov puros y que el cruce Merino D'Arles $x$ Romanov (3); esta resistencia a los endoparásitos también fue evidenciada en ovejas de la raza nativa de La Florida, St. Croix y en la Barbados barriga negra con respecto al cruce Dorset $x$ Rambouillet (5).

Sin embargo, es importante destacar que en una misma raza existe también una significativa variación de dicha resistencia, la cual puede incluso ser de la misma magnitud que la observada entre razas $(3,4,6,7)$.
En conclusión, la resistencia a la infección parasitaria es variable tanto entre razas como en el interior de ellas, y es de naturaleza genética $y$, por consiguiente, heredable $(3,4,6,7,8)$. El hecho de que la resistencia a la infección por estróngilos digestivos sea heredable permite implementar programas de selección de ovinos, incluyendo el criterio "resistencia a la infección parasitaria", lo cual está aún más justificado si consideramos los resultados de Gray (7), quien estableció que cuando los ovinos son apareados considerando el criterio resistencia a la infección parasitaria, su productividad no declina; a lo cual se puede agregar, que el uso de animales helmintorresistentes mejora la calidad de los suelos, en vista de que limitar la frecuencia del uso de antihelmínticos favorece el desarrollo y la supervivencia de la fauna edáfica (lombrices de tierra, escarabajos), que son afectados negativamente por los residuos de algunos antiparasitarios de amplio espectro, como las Ivermectinas, presentes en las heces de los animales tratados. La conservación de esta fauna edáfica es de gran interés, ya que al desagregar la materia fecal y enterrar las formas de diseminación de los estróngilos digestivos esta realiza un control biológico que se traduce en disminución de la contaminación del pastizal (2).

El objetivo del presente trabajo es evaluar la resistencia de los padrotes a la infección por estróngilos digestivos, expresada mediante los niveles de infección de ellos mismos y los de sus hijas, como criterio para seleccionar padrotes helmintorresistentes.

\section{MATERIALES Y MÉTODOS}

Lugar de los ensayos. Se logró la colaboración de los propietarios de tres pequeñas unidades de producción ubicadas en el municipio José Antonio Páez, del estado Yaracuy (Venezuela). Las tres explotaciones son mixtas (bovino-ovino).

- Granja A: El rebaño ovino está compuesto por 45 animales, con hembras mestizas, y el reproductor es de la raza Bergamasca; tiene un corral como instalación de resguardo.

- Granja B: El rebaño ovino está compuesto por 23 animales, con hembras mestizas, y el reproductor 
es de la raza Barbados Barriga Negra (BBN 1); tiene un aprisco para el resguardo de los animales.

- Granja C: El rebaño está constituido por 80 animales, con hembras mestizas, y el reproductor es de la raza Barbados Barriga Negra (BBN 2); como instalación de resguardo tiene un corral.

En las tres explotaciones las hembras madres eran mestizas de West African o Persa Cabeza Negra.

Selección de las corderas del ensayo. En cada explotación se seleccionó un reproductor y 10 de sus hijas, todas provenientes de madres mestizas de West African o Persa Cabeza Negra. En todos los casos, las hembras tenían al comienzo del ensayo edades de 3 a 4 meses, aproximadamente. Con la finalidad de obtener información de animales a pastoreo, comenzando en la etapa de mayor susceptibilidad, y con los muestreos posteriores poder definir en cuáles descendientes la expresión de resistencia se manifiesta en bajos o nulos recuentos de huevos por gramo de heces (hpg), se realizaron muestreos coprológicos durante 12 quincenas sucesivas. A los padrotes se les tomaron muestras de heces el mismo día del muestreo de sus hijas.

Cada hembra del ensayo fue identificada mediante un tatuaje en la oreja derecha. Dos de los padrotes cuyas hijas formaron parte del presente ensayo eran de la raza Barbados Barriga Negra (BBN 1 y BBN 2), y el otro era Bergamasca. Previo al inicio del ensayo, todos los animales seleccionados fueron pesados y tratados con Albendazol $^{\circledR}$ a la

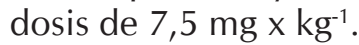

Alimentación. Los propietarios de los tres rebaños ovinos trasladan diariamente a sus animales a áreas comunales o de poscosecha, en las cuales se utilizan los recursos naturales existentes, tales como gramíneas, leguminosas de bajo porte, hojas, vainas, así como la soca de cultivos como maíz, caraota, fríjol, etc., lo que se corresponde con un sistema silvopastoril multiestrato. Los animales no reciben ningún tipo de suplementación mineral ni concentrados.

Métodos parasitológicos. Según Gogolin et al. (9), uno de los mayores problemas en la selección con base en la resistencia a la infección parasitaria ha sido la escogencia de los mejores criterios por emplear. Es indiscutible que la mejor evaluación para caracterizar el nivel de resistencia lo constituye la carga parasitaria (número de vermes instalados en el tracto gastrointestinal), pero la obtención de esta información solo es posible después de una necropsia parasitaria, lo cual, obviamente, no es factible como criterio de selección individual; esto lleva a la escogencia de los exámenes coproscópicos cuantitativos, cuyos resultados, expresados en número de huevos por gramo de heces (hpg), han sido de gran utilidad para evaluar la resistencia o la susceptibilidad tanto dentro de una raza como entre razas y cepas (4). Por otra parte, la cuantificación de los hpg individualizados nos aporta información sobre el poder contaminante para el pastizal de un animal determinado. Para el desarrollo del presente trabajo se realizaron las siguientes labores:

Durante doce quincenas consecutivas se tomaron muestras de heces directamente del recto de cada animal, se identificaron y se trasladaron al laboratorio, bajo refrigeración, para su procesamiento inmediato o en un lapso no mayor de 48 horas. La técnica empleada fue la de McMaster (10), y como líquido de flotación se utilizó una solución sobresaturada de NACl. Considerando la importancia del recuento de huevos de estróngilos digestivos por gramo de heces, tanto para evaluar la carga parasitaria del animal como su poder contaminador del pastizal, se establecieron los siguientes valores:

Tabla I. Valores de carga parasitaria establecidos para el desarrollo de la investigación

\begin{tabular}{|c|c|}
\hline Animales & Recuento hpg \\
\hline Negativos & 0 \\
\hline Leves & $50-150$ \\
\hline Moderados & $200-700$ \\
\hline Altas & $>750$ \\
\hline
\end{tabular}

Análisis de los datos. Previo al análisis de los datos se realizó la categorización de los recuentos de hpg durante los 15 muestreos consecutivos, considerando los valores de ubicación de cada hija en cada muestreo de acuerdo con su recuento de hpg, como Negativa, Leve, Moderada o Alta, utilizando para ello la escala suministrada en 
métodos parasitológicos. Luego, la información categorizada fue organizada en una tabla de contingencia múltiple $(3 \times 4)$ entre dicha clasificación parasitológica y el padre de cada lote de hembras, correspondiéndoles a los padres la ubicación en las filas, y los niveles de infección de las hijas categorizadas en las columnas. En el caso de los padrotes se realizó la categorización de los recuentos de hpg de la misma forma que se hizo con las corderas, y se construyó una tabla de contingencia de $3 \times 4$, colocando los padrotes en las filas, y sus niveles de infección categorizados en las columnas. En este caso, y previo a la conformación de la tabla, se realizó un remuestreo aleatorio con reposición, lo cual nos permitió obtener desde el conjunto de datos base $(n=12$ por padrote) muestras con un tamaño distinto y superior ( $n=120$ por padrote) (11).
Con la información contenida en dicha tabla se realizó un análisis de correspondencia simple, para lo cual empleamos el paquete estadístico InfoStat (2004).

\section{RESULTADOS Y DISCUSIÓN}

En la Figura 1 se observa que el padrote de raza Bergamasca se asoció con hijas con niveles de infección leves o moderadas, mientras que uno de los padrotes de la raza Barbados Barriga Negra, el BBN 1, se asoció con hijas negativas a la infección por estróngilos digestivos, y el otro (BBN 2) mostró asociación con niveles de infección alta de sus hijas.

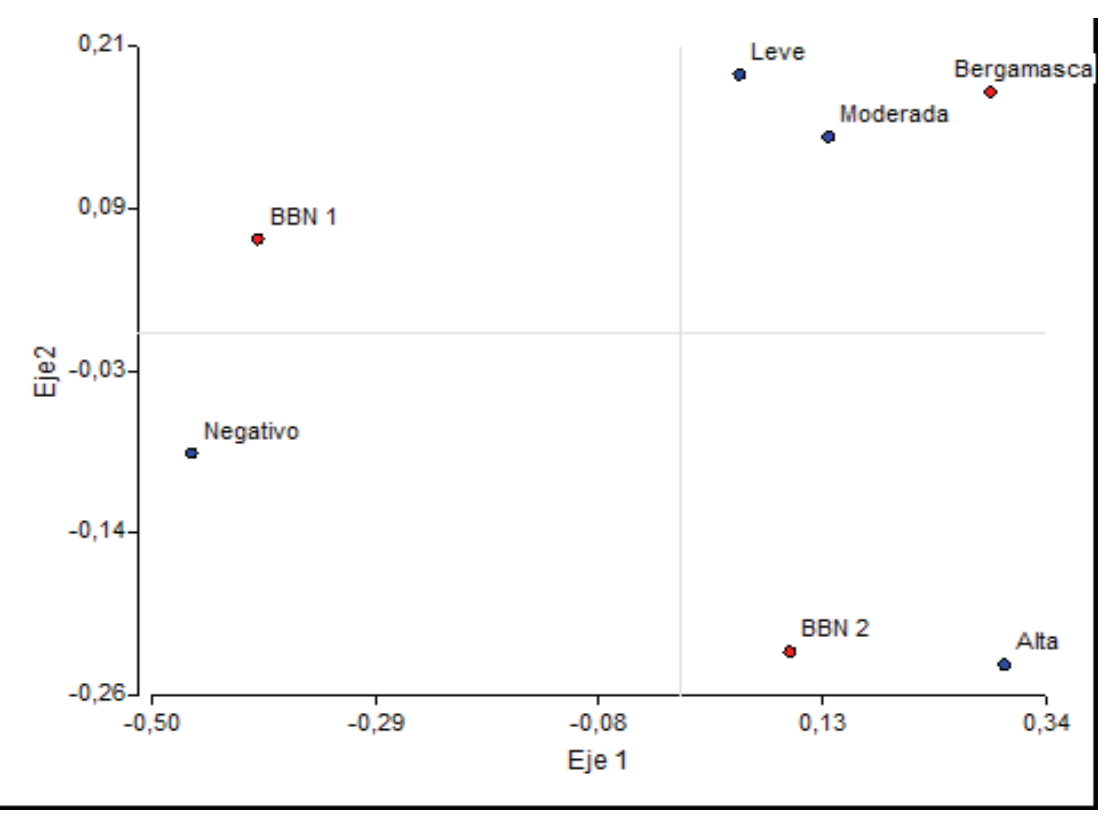

Figura 1. Análisis de correspondencia entre los niveles de infección de corderas y sus respectivos padres (BBN $1=$ Barbados Barriga Negra 1 y BBN $2=$ Barbados Barriga Negra 2)

En la Figura 2, en la cual se graficó cada padrote con sus respectivos niveles de infección, vemos que el padrote Bergamasca se asocia con niveles de infección moderados; el Barbados Barriga Negra 1
(BBN 1), con niveles de infección que oscilan entre leve y moderado, y el padrote Barbados Barriga Negra 2 (BBN 2), con niveles de infección altos. 


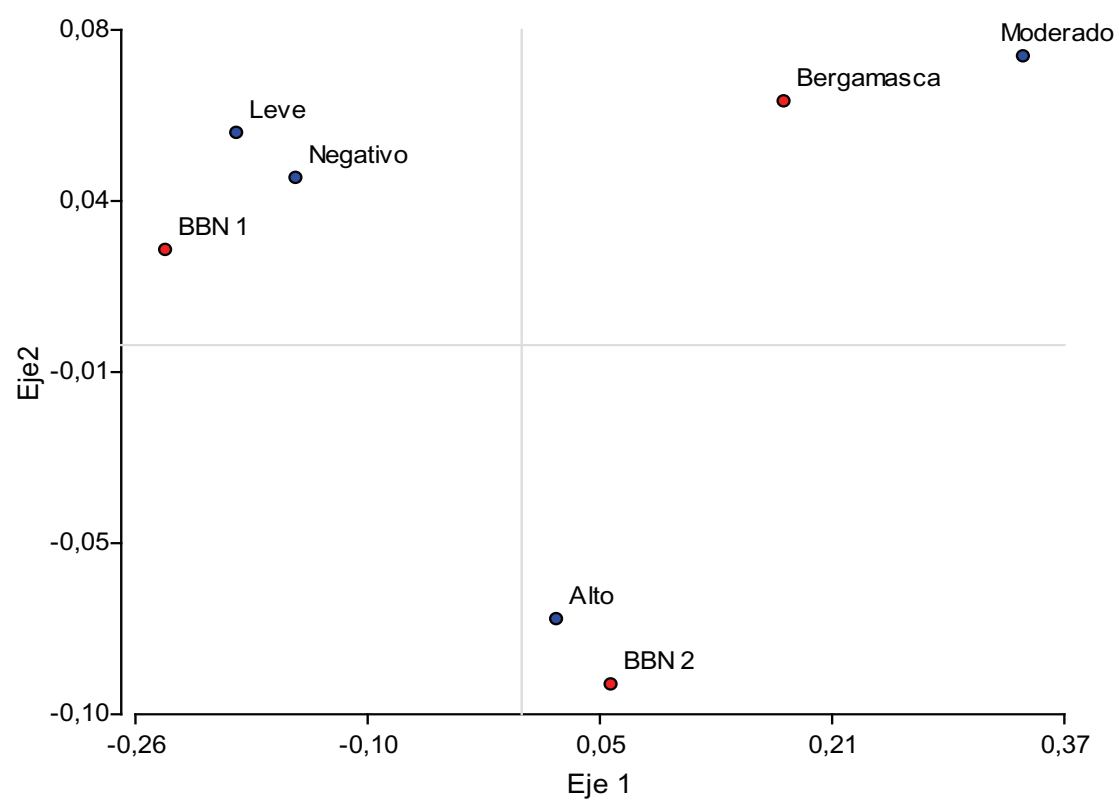

Figura 2. Análisis de correspondencia entre los niveles de infección y la raza de los padrotes (BBN 1 = Barbados Barriga Negra 1 y BBN 2 = Barbados Barriga Negra 2)

Para Lejambre (8), el parasitismo ocasionado por nemátodos gastrointestinales, en particular por los de la familia Trichostrongylidae, es el resultado de la interacción entre el genotipo del hospedador y el de las poblaciones parasitarias, así como del medioambiente común compartido por el hospedador y los parásitos, destacando el hecho de que aun en animales pertenecientes al mismo rebaño, e incluso a la misma raza, sexo, condición fisiológica y edad, los niveles de infección no son homogéneos $(2,3,12)$.

Por otra parte, existen abundantes reportes sobre la cada vez más frecuente quimiorresistencia de cepas de nemátodos gastrointestinales de rumiantes $(10,13,14)$, situación que se agrava debido a las pocas alternativas eficaces frente al empleo de quimioterápicos en el control parasitario (1), lo cual se complica debido a la resistencia cruzada entre muchas de las moléculas que se consiguen en el mercado $(3,15)$. Lo anteriormente expuesto constituye una de las principales justificaciones de ensayos que conduzcan a la obtención de animales helmintorresistentes, proceso en el cual la selección de padrotes y de vientres, considerando su capacidad de resistencia a la infección parasitaria, y su utilización en programas de cruzamiento posibilitan la obtención de descendientes helmintorresistentes, dado el carácter hereditario de dicha condición (4). Nuestros resultados, aunque limitados por la poca cantidad de animales empleados (3 padrotes y 30 corderas), evidenciaron que uno de los padrotes de raza Barbados Barriga Negra, el BBN 2, se asoció con cargas parasitarias elevadas, y que sus hijas también se asociaron con niveles altos de infección. En el caso del padrote de raza Bergamasca, su asociación fue con niveles de infección moderados, y sus hijas, con niveles leves o moderados. El otro padrote de raza Barbados Barriga Negra, el BBN 1, se asoció con infecciones leves y moderadas, pero sus hijas se asociaron con la condición de negativas. La anterior es una información de enorme importancia para el productor, ya que, sin duda, aquellos padrotes cuya descendencia se asocie con niveles de infección altos no deberían ser conservados como reproductores. Estos resultados destacan la importancia de la evaluación tanto de los padrotes como de los futuros vientres, debido a la enorme heterogeneidad frente a la infección parasitaria que puede observarse no solo entre razas, sino en el interior de una misma raza $(3,4)$. 
Nivel de infección por estróngilos digestivos de padrotes ovinos y de sus hijas como criterio de selección de ovinos helmintorresistentes

\section{CONCLUSIONES}

Estas evaluaciones requieren de un laboratorio de helmintología con equipamiento básico, ya que mediante técnicas sencillas de coproscopía cuantitativa, realizadas en forma periódica, podemos discriminar en el interior de un rebaño los animales en las categorías de acuerdo con sus niveles de infección y la evaluación simultánea de los padrotes, y un lote de sus respectivas hijas constituirán la base para la escogencia del padrote y de los futuros vientres, que además de cumplir con los requisitos zootécnicos tengan el valor agregado de ser helmintorresistentes, lo cual permitiría mejorar la rentabilidad de las explotaciones ovinas al disminuir la mortalidad por las infecciones parasitarias y por la menor frecuencia en el uso de antihelmínticos.

El análisis multivariado de correspondencia lineal múltiple se revela de gran utilidad para establecer la correspondencia de padres e hijas con sus respectivos niveles de infección por nemátodos gastroentéricos, y, por consiguiente, de gran ayuda en programas de control parasitario que contemplen el uso de ovinos helmintorresistentes.

\section{REFERENCIAS}

(1) FAO. Resistencia a los antiparasitarios: Estado actual con énfasis en América Latina. Dirección de Producción y Salud Animal. Roma, 52 pp. 2003.

(2) Morales CG, Sandoval E, Pino L, Jiménez D. Utilización derumiantes domésticos genéticamente resistentes a la infección por estróngilos digestivos en estrategias de control. Revista Digital CENIAP (en línea), 2005; 8: 46-53. Disponible en http// www.ceniap.gov.ve/ceniaphoy/articulos/n8/arti/ morales_g1/morales_g1.htm

(3) Mandonnet N. Analyse de la variabilité génétique de la resístance aux strongles gastrointestinaux chez les petits ruminants. Elements pour la definition d'objetifs et de critéres de sélection en milieu tempéré ou tropical .These Docteur en Sciences. Orsay, Paris; Université de Paris XI, 1995.

(4) Baker R. Genetic resistance to endoparasites in sheep and goats in the tropics and evidence for resistance in some sheep and goats breeds in subhumid coastal Kenya. Animal Genetic Resources Information 2009; 24:13-30

(5) Courtney C, Parker C, McClure K, Herd R. Ressistance of exotic and domestic lambs to experimental infection with Haemonchus contortus. International Journal for Parasitology, 1985; 15 (1): 101-109.

(6) Stear M, Murray M. Genetic resistance to parasitic disease: particularly of resistance in ruminants to gastrointestinal nematodes. Veterinary Parasitology 1994; 54: 161-176.

(7) Gray G. The use of genetically resistant sheep to control nematode parasitism. Veterinary Parasitology 1997; 72: 345-366.

(8) Lejambre L. Genetic and the control of trichostrongylid parasites of ruminants. In: Biology and control of endoparasites. Australia: Academic Press: 53-80. 1982.

(9) Gogolin K, Maddox J, Fabb S, Brandom M. Genetic markers for the selection of parasite resistance in livestock in animal parasite control utilizing biotechnology. Edited by: W.K. Yong; C.R.C Press, U.S.A: 407-456. 1992.

(10) Morales G, Pino LA. Nemátodos, parásitos de los rumiantes domésticos en Venezuela: diagnóstico y control. Dot Print C. A; Caracas; 143, pp. 2009.

(11) InfoStat. InfoStat versión 2004. Grupo InfoStat, FCA, Universidad Nacional de Córdoba, Argentina. 2004.

(12) Morales G, Pino LA, Sandoval E, Jiménez D, Morales J. Relación entre la condición corporal y el nivel de infestación parasitaria en bovinos a pastoreo como criterio para el tratamiento antihelmíntico selectivo. Rev. Inv. Vet. Perú, 2012; 23(1): 80-89.

(13) Waller P. Anthelmintic resistance. Veterinary Parasitology 1997; 72: 391-412.

(14) Pritchard R. Anthelmintic resistance. Veterinary Parasitology 1994; 54: 259-268.

(15) Urquhart G, Armour J, Duncan J, Dunn A, Jennigs F. Veterinary Parasitology. Blackwell Science, $2^{\text {nd }}$. Edition, United Kingdom, 307, pp. 1999. 